\title{
The Role of SARS-CoV-2 Etiotropic Therapy in Controlling the Spread of Infection
}

\section{Khavkina DA ${ }^{1}$, Chukhliaev $\mathrm{PV}^{1}$ and Ruzhentsova TA ${ }^{2 *}$}

${ }^{1}$ Junior Researcher of the Clinical Department, Gabrichevsky research institute for Epidemiology and Microbiology, Russia

${ }^{2}$ Deputy Director of the Clinical Department, Gabrichevsky research institute for Epidemiology and Microbiology, Russia

*Corresponding author: Tatiana A Ruzhentsova, Doctor of medical sciences, Deputy Director of the Clinical Department of the Gabrichevsky research institute for epidemiology and microbiology, Russian Federal Supervision Service for Consumer Rights Protection and People’s Welfare, 125212, Moscow, Russia, Tel: +74953045696; Email: ruzhencova@gmail.com

\section{Abstract}

Introduction: The COVID-19 pandemic is forcing epidemiologists to look for new ways to stop the spread of infection. The article presents the results of a study in patients with mild to moderate COVID-19. The study included 244 patients aged $18-90$ years with confirmed SARS-CoV-2 infection (by PCR of oropharyngeal and nasopharyngeal smears).

Materials and Methods: Among them, at the time of inclusion in the study and the beginning of therapy, 203 patients were treated on an outpatient basis, and 41 - in a hospital. Significant differences were found in the time to virus elimination in the compared groups among all patients.

Results: Thus, a median of 8.5 days was established in the group of patients who did not take antiviral therapy, compared with groups 1 and 2, where the median was 3 days. However, among hospitalized patients, virus elimination was significantly faster when taking favipiravir: 3.0 compared to 5.0 days in the comparison group the comparison group that received umifenovir ( $\mathrm{p}$ =0.038).

Conclusion: The use of effective antiviral therapy leads to a reduction in the time of elimination of the virus, which is a key process in interrupting its further transmission.

Keywords: Virus Elimination; SARS-CoV-2; COVID-19; Infection

\section{Abbreviations: M: Median; IQR: Interquartile Range.}

\section{Introduction}

Since the epidemic outbreak of COVID-19 in Wuhan (China), the search for optimal treatment of the disease has not stopped. During this time, various schemes were tested: hydroxychloroquine, monoclonal antibodies, convalescent plasma. None of them gave the main expected effect - the fastest possible elimination of the virus. The use of lopinavir, ritonavir also did not meet expectations [1-4]. At the same time, there was more and more information about the ways of transmission of the virus. It is known that SARS-CoV-2 can be transmitted by contact and airborne droplets. Unconfirmed, but very disturbing, reports of possible fecal-oral and sexual transmission of the infection began to appear. 


\section{Epidemiology International Journal}

While clinicians around the world fought to save patients with COVID-19, epidemiologists were focused on finding a drug that could break the vicious cycle of the epidemic process, interrupting the transmission of infection from carriers and infected people to healthy people. Only rapid elimination can help reduce the number of infected people and stop the spread of the pandemic. By the end of the first wave, the need for backup antiviral drugs, such as favipiravir, became apparent. Despite the presence of teratogenic effects and a number of adverse reactions, its use in comparison with umifenovir, hydroxychloroquine and standard therapy without the use of antiviral agents showed greater effectiveness in the rate of elimination of the virus, especially in the initial phase of the disease [5].

\section{Objective}

To evaluate the rate of elimination of SARS-CoV-2 with and without antiviral therapy.

\section{Materials and Methods}

A clinical study was conducted to assess the elimination rate of SARS-CoV-2 in patients with mild to moderate COVID-19. The study included 244 patients aged 18-90 years with confirmed SARS-CoV-2 infection (by PCR of oropharyngeal and nasopharyngeal smears). Among them, at the time of inclusion in the study and the beginning of therapy, 203 patients were treated on an outpatient basis, and 41 - in a hospital. Patients were randomized in a 3:1 ratio. Group 1 received favipiravir $1800 \mathrm{mg}$ twice daily on day 1 , then: from day 2 to day $10-800 \mathrm{mg}$ twice daily). The second group received other etiotropic therapy: outpatient cohort received umifenovir + intranasal interferon alpha-2b, and the hospitalized patients received hydroxychloroquine for up to 10 days. The third group received azithromycin and vitamins, without the inclusion of antiviral agents. All patients received the necessary concomitant symptomatic therapy. The median (M) and interquartile range (IQR) estimate of the virus elimination period was taken as the primary endpoint. The Kaplan-Meier method was used to estimate the primary endpoints time to viral clearance. The log-rank test was used to assess between-group differences in these endpoints.

\section{Results}

Significant differences were found in the time to virus elimination in the compared groups among all patients. Thus, a median of 8.5 days was established in the group of patients who did not take antiviral therapy, compared with groups 1 and 2, where the median was 3 days. However, among hospitalized patients, virus elimination was significantly faster when taking favipiravir: 3.0 compared to 5.0 days in the comparison group received hydroxychloroquine $(\mathrm{p}=0.038$, Table 1). The virus elimination rate was statistically significantly higher on days 3 and 5 in group 1 with favipiravir compared to group 2 with standard therapy: on day $3(p=0.030)$, on day $5(p=0.022)$, and group 3-on day $8.5(\mathrm{p}<0.05)$.

\begin{tabular}{|c|c|c|c|c|}
\hline \multirow{3}{*}{ Indicator } & Favipiravir, & $\begin{array}{c}\text { Other etiotropic } \\
\text { therapy, } n=56 \text {, }\end{array}$ & $\begin{array}{c}\text { Therapy without the use } \\
\text { of antiviral agents, }\end{array}$ & \multirow{3}{*}{ Meaning (p) } \\
\hline & $\mathrm{n}=112$, & M (IQR) & $\mathrm{n}==\mathbf{7 6}$ & \\
\hline & M (IQR) & & M (IQR) & \\
\hline \multicolumn{5}{|c|}{ Virus elimination time } \\
\hline $\begin{array}{l}\text { The entire patient } \\
\text { population }\end{array}$ & $3,0(3,0-3,5)$ & $3,0(3,0-7,0)$ & $8,5(7,0-10,0)$ & 0,523 \\
\hline Outpatient & \multirow{2}{*}{$3,0(3,0-5,0)$} & \multirow{2}{*}{$3,0(3,0-7,0)$} & \multirow{2}{*}{$8,5(7,0-10,0)$} & \multirow{2}{*}{0,450} \\
\hline cohort & & & & \\
\hline Hospital cohort & $3,0(3,0-3,0)$ & $5,0(4,5-5,5)$ & - & 0,029 \\
\hline
\end{tabular}

Table 1: Virus elimination time in the compared groups.

The frequency of adverse therapy outcomes was noted in group 3, and was clearly associated with the duration of virus isolation. Of the 76 patients in group 3,11 patients went from moderate-severe to extremely severe during treatment, and 4 deaths were noted, in which the duration of isolation ranged from 16 to 35 days. It should also be noted that among the lethal patients, despair therapy was actively used against the background of the absence of antiviral accompaniment or the use of dexamethasone, monoclonal antibody drugs as antiviral therapy.

\section{Discussion}

The laws of the epidemic process are relevant for any infection, in this regard; SARS-CoV-2 is no exception: interrupting the transmission chain is a key link in the fight 


\section{Epidemiology International Journal}

against a pandemic. The study showed that achieving this goal is possible with the use of effective antiviral therapy. It is obvious that during the detection of the virus on the nasopharyngeal mucosa, the patient continues to secrete it into the environment. At the same time, the risk remains of transmitting the pathogen to family members, neighbors, and medical personnel. It is impossible to completely exclude the possibility of spreading the infection to neighboring premises, when delivering food and essential goods even if the recommendations for isolation of the patient are strictly followed. Thus, reducing the persistence period of the virus can reduce the intensity of the spread of infection.

\section{Conclusion}

The use of effective antiviral therapy leads to a reduction in the time of elimination of the virus, which is a key process in interrupting its further transmission.

\section{References}

1. Yao X, Ye F, Zhang M, Cui C, Huang B, et al. (2020) In Vitro Antiviral Activity and Projection of Optimized
Dosing Design of Hydroxychloroquine for the Treatment of Severe Acute Respiratory Syndrome Coronavirus 2 (SARS-CoV-2). Clin Infect Dis 71(15): 732-739.

2. Gautret P, Lagier JC, Parola P, Hoang VT, Meddeb L, et al. (2020) Hydroxychloroquine and azithromycin as a treatment of COVID?19: results of an open-label nonrandomized clinical trial. Int J Antimicrob Agents 56(1): 105949.

3. Chan JF, Yao Y, Yeung ML, Deng W, Bao L, et al. (2015) Treatment With Lopinavir/Ritonavir or Interferon?1b Improves Outcome of MERS-CoV Infection in a Nonhuman Primate Model of Common Marmoset. J Infect Dis 212(12): 1904-1913.

4. Cao B, Wang Y, Wen D, Liu W, Wang J, et al. (2020) A Trial of Lopinavir-Ritonavir in Adults Hospitalized with Severe Covid-19. N Engl J Med 382(19): 1787-1799.

5. Ruzhentsova TA, Chukhliaev PV, Khavkina DA, Garbuzov A, Oseshnyuk R, et al. (2020) Phase 3 Trial of Coronavir (Favipiravir) in Patients with Mild to Moderate COVID-19. SSRN. 